\title{
A NEW TYPE OF SINGLE-MODE LMA PHOTONIC CRYSTAL FIBER BASED ON INDEX-MATCHING COUPLING
}

\author{
Tanmay Bhowmik ${ }^{1 *}$, Annesha Maity ${ }^{2}$ \\ ${ }^{*}$ M.Tech Student (PG Research), Dept. of Electronics \& Communication Engineering, Heritage Institute of \\ Technology (HIT),Kolkata, West Bengal, India. \\ tanmay.alums@gmail.com \\ ${ }^{2}$ Assistant Professor, Dept. of Electronics \& Communication Engineering, Heritage Institute of Technology \\ (HIT),Kolkata, West Bengal, India
}

\begin{abstract}
In this paper, new type wideband large mode area (LMA), mono-mode holey fiber or photonic crystal fiber $(P C F)$ based on indexmatching resonant-coupling is proposed by using the FEM (finite-element method). The novel design has been achieved for a wide wavelength froml.5 $\mu \mathrm{m}$ to $1.63 \mu \mathrm{m}$. Thus, the total operation bandwidth reaches $130 \mathrm{~nm}$. The higher order mode resonant coupling loss of the proposed fiber is good enough within this wavelength range to maintain the mono-mode operation. The total effective area for the proposed fiber is larger than $2150 \mu \mathrm{m}^{2}$. The proposed fiber exhibits a very less FM (fundamental mode or LPO mode) loss about $10^{-3} \mathrm{~dB} / \mathrm{m}$ and the HOM (higher order mode) resonant coupling loss is larger than $6.8 \mathrm{~dB} / \mathrm{m}$ at $1.55 \mu \mathrm{m}$. The large mode area can reduce optical intensities and thus the fiber design can gain more attraction in the field of fiber laser and amplifier based applications. Moreover, the broadband design which covers the key wavelength (1550 $\mathrm{nm}$ ) for communication window, will pay attention to the manufacturer in the fiber optic communication.
\end{abstract}

Keywords: Index-Matching Resonant-Coupling, Higher Order Mode Suppression, LMA (Large Mode Area) Fiber, Mono-Mode, Photonic Crystal Fiber (PCF).

\section{INTRODUCTION}

Optical fiber technology revolutionised telecommunication in the late twentieth century, and photonic crystal fiber(PCF) now stands to revolutionise optical fiber technology itself. Aphotonic crystal fiber (PCF) is a micro-structured fiber where the core light is confined by an arrangement of air holes in the cladding structure and the core is formed by one or more missing air holes in the middle of the fiber. One or more missing hole in the air hole array forms a section which effectively has a superior index of refraction than that of the surrounding photonic crystal. For this phenomena, it acts as a waveguide core where light can become trapped and forming a guided mode [1]. Photonic crystal fibers or microstructured holey-fibers have all potential to get all kind of attention only because these kind of structure can provide an unprecedented degree of freedom in tailoring their modal properties. However, there are numerous challenges still remain in case of the nonlinearity of the fiber being induced for the present of high power. The rod-type fibers or PCF with a large mode area provide the fruitful resolution to the issue as the nonlinearity of fiber is inversely proportional to MFA ("mode field area") or effective mode area [2].

For the waned optical intensities, these kind of fibers have lesser nonlinearities and the much greater damage-threshold and thus they are more suitable in the intense-pulses amplification or the signals having single-frequency in optical fiber amplifiers. The standard mono-mode fibers have the MFA or effective area less than $90 \mu \mathrm{m}^{2}$, whereas the LMA fibers can have thousands of $\mu \mathrm{m}^{2}$.

On the other hand, to provide great beam quality as well as the stability of the output given by lasers, the very important property for such LMA type fibers is to maintain the single mode operation. Moreover, low loss FM (LP01 mode) propagation and high loss of the higher order mode (LP11 mode) propagation are the two key requirements for fibers to be useful in efficient high power systems [3].

The proposed design provides all such improvised properties mentioned above including a novel and exciting design based on "resonant-coupling higher-order-mode (HOM) suppression" and the design also provides a large "mode field area" (MFA) about $2200 \mu \mathrm{m}^{2}$.

The rest of this paper is formed as follows. The section-2 gives all the basic and useful concepts which help to understand the fiber design thoroughly and also shows that the "higher order mode suppression" in the microstructured fibers can be narrated by the basic mechanism of "index matching resonant coupling" between core higher order modes and cladding modes. Moreover, this primitive mechanism has been audited by several researchers [4-6]. We thereafter take this affect and thus propose a general novel design principles for HOM-suppressed LMA-fiber by using the finite element method (FEM). The structure is then gradually optimised and explained in subsequent sections. In Section-3, the fiber design parameters have been described. 
Section-4 elaborates the methodology of the fiber design. Section-5 shows how the basic structure optimised towards a wideband of operation and the results are also shown respectively. The results are also summarized in this section. Finally, section 6 concludes this paper.

The PCF operation band is achieved for $1.50 \mu \mathrm{m}$ to $1.63 \mu \mathrm{m}$ wavelength range respectively. This wavelength region has the key window for communication application $(1550 \mathrm{~nm})$. On the other side, "large mode area" and low "HOMconfinement loss" altogether make the fiber of a supreme potential in the fiber-lasers.

\section{LMA PCFPRINCIPLESAND PROPERTIES}

As per the guidance-mechanism, the "photonic crystal fibers" can be classified into 2 categories [7]:

- Firstly "Index-guiding fibers" with the "total internal reflection or TIR". This has also termed as a solid-core fiber.

- And second, "photonic band gap (PBG)fiber". In this case, the fiber has a central air hole and the light is confined to it. This has also termed as hollow core fiber.

In the subsequent sections, the index-guiding fibers are considered only. The "index-guiding fibers" or "solid core fibers" are characterized by an average index of refraction of the core area higher than the cladding region. Moreover, LMA fiber is the most attractive PCF.

\subsection{Index Guiding Fibers}

In case ofthe conventional fiber, the total number of the bound-modes can be determined by the V-number. This parameter increases along with the decrease of wavelength. The "V parameter"also can define for an index-guiding LMA fiber as the same way as that of a step index fiber with the following equations. Mortensen et al. had given a basic formulation of the "V" parameter as follows: [8]

$$
\begin{aligned}
& V_{\text {Step }}=\frac{2 \pi}{\lambda} r\left(n_{\text {Core }}^{2}(\lambda)-n_{\text {Cladding }}^{2}(\lambda)\right)^{1 / 2} \\
& V_{P C F}=\frac{2 \pi}{\lambda} \Lambda\left(n_{F M}^{2}(\lambda)-n_{f s m}^{2}(\lambda)\right)^{1 / 2}
\end{aligned}
$$

Though this mathematical expression is almost same as that form which is known for standard fibers, but the unique natures of the holey-fiber or photonic crystal fibers are taken into account. From equation $(2),(\Lambda)$ is the fiber pitch (distance between the centers of the air holes), " $n_{F M}(\lambda)$ " is the effective index of refraction of the FM (fundamental mode), which is wavelength dependent and $n_{f s m}(\lambda)$ refers the corresponding index of refraction of the cladding mode also often termed as the FSM (fundamental space filling mode) [9].

For the fiber to operate in the mono-mode, the V-parameter has to be less than 2.405 at any optical wavelengths. Thus, mono-mode type fibers can be multimoded for the light that has sufficiently shorter wavelength (w.r.t the radius of the cavities). Russell et al. proposedauseful explanation towards the mono-mode property of the microstructured fibers based on that of the effective index of refraction of the cladding region [10].

PCFs thus can be engineered to guide only the FM, and also can perform under SM-operation over all of the optical wavelengths if $d / \Lambda<0.407$. For the purpose of investigation of the mono-mode condition, the FM (fundamental mode)has to be solved for the wide region of wavelengths.

\subsection{Approach to Large-Mode-Area (LMA) PCF}

The Optical-transmission system requiresphotonic crystal fibers which have a large MFA (mode field area) [11]. This type of fiber is required for various purpose e.g. to support wideband optical transmissions, to the purpose of minimizing the coupling-loss to that of the standard "single mode fibers (SMFs)". Moreover, in case of wideband communication-systems, confinement losses of the FMs and the HOMsplaythe vital roles. As an example, in case of the WDM (wavelength division multiplexing) communication systems, the essential part is to maintain and provide a uniform response for the different channels of wavelengths. Since the HOMs (higher order modes) suffer a large propagation-loss and on the other hand the FM (fundamental mode) guided with a very lower propagation loss, this kind of fiber is said to be operate as mono-mode or single mode (SM).

The benefits of the large mode area holey- fiber or PCF are-

- Mono-mode operation by HOM suppression;

- Can handle the very high average and peak power,

- Provides lesser nonlinearities,

- Fiber loss is marginable low,

- The diameter of the mode field can be independent of wavelength.

Applications of the LMA fibers are delivery of high power, delivery of short pulse, filtering of mode, laser pig-tailing, guidance of multiwavelengths, wideband interferometry. For the higher value of pitch effective mode area is also increased and again while the area is increased, the more light will pass through the core region.

The improved SM-properties can achieve by delocalizing the HOMs in the core region as well as by providing preferential gain for that of the FM. Thus, such a large core can be operated at significantly for powers of high peak as well as for the pulse energies without to reach nonlinear effect threshold levels. Thus, this structure results in high mode-discrimination with only a degradation of moderate beam quality that of the FM only. The LMA design is therefore proven to work in high power operation as well as provides SM-operation too.

Due to the lobe dimension of " $2 \Lambda$ ", the FM or "fundamental transverse mode" perfectly fits into the core region. 
On the other hand, HOMs have much lesser transverse lobe dimensions so that can leak away from the core through gaps between the air holes. When the diameter of that air holes is increased, gaps between them become smaller and smaller, thus less HOMs (higher order modes) can leak away from the core region and thus therefore it is possible to confine more higher order modes in the core region. Hence, PCFs with a $d / \Lambda>0.45$ can be multimoded.

\subsection{Nonlinear Coefficient and Mode Field Area}

The most of the nonlinear-effects in case of fiber-optic propagation raise from that of the nonlinear refraction.

With a higher nonlinearity in holey fibers, the propagation loss is also increased very quickly. Although, such holeyfibers aren't used in case of long-haul communication.

So, the minimization of the nonlinearity in holey- fibers is quite necessary. The Large mode field area (MFA) of the holey- fibers can prevent the unwanted nonlinear breaches. Moreover, lower confinement losses or small MFA is also might require for the some other special applications. The nonlinear-coefficient, " $\gamma$ " in $W^{-1} \mathrm{Km}^{-1}$ can calculate by the following formula [12],

$$
\gamma=\frac{2 \pi n_{2}}{\lambda * A_{e f f}}
$$

Where $n_{2}$ refers the nonlinear index of refraction of silica $\left(2.66 \times 10^{-20} \mathrm{~m}^{2} / W\right)$ and $A_{\text {eff }}$ is the total effective area. Thus, nonlinear-coefficient is the most important and notable parameter in the case of the nonlinearities. Again, it also has a connection to that of the leakage loss and NA (numerical aperture). For a nonlinear measurement, the lower MFA or effective mode area provides a higher density of power and again the effects produced by nonlinearity are dependent to the intensities of the EMF ("electromagnetic field"). The intensity I, over the core area $A_{\text {eff }}$ can be related with the power, $P_{\text {mean }}$ and can be defined as

$$
I=\frac{P_{\text {mean }}}{A_{\text {eff }}}
$$

The MFA or effective area is another primary key factor in the designing of PCFs.The MFA or effective mode area is calculated by [12],

$$
A_{e f f}=\frac{\left[\iint_{S}|E(x ; y)|^{2} d x . d y\right]^{2}}{\iint_{S}|E(x ; y)|^{4} d x . d y}
$$

Here; $\mathrm{E}(\mathrm{x} ; \mathrm{y})$ refers the vector quantity of the transverse electric field and the " $S$ "represents the cross section of whole fiber.

As we can see in equation-(4); the non-linear effects are inversely proportional to that of the mode field are $\left(A_{e f f}\right)$.
Thus; the large mode area fibers (LMA-fibers) can introduce up-scaling of delivered power and can be a more important feature in case of the fiber optic communication.

\subsection{Confinement Loss}

If an EM wave is propagating through a microstructured holeyfiber,amore or less whack of the energy definitely releases. The confinement loss is another form of leaking of energy that happens in fibers with single material as the connately leaky nature provided by the guided modes within these type of fibers. The whole phenomenon occurs because of the core-index is same as that of the index of the outer cladding.

Due to the air holes number is finite, the leakage of power is unpreventable. Therefore, one must careful in selection of the parameters like hole-diameters $(d)$ and that of pitch $(\Lambda)$ so that the minimization of the loss can possible as per as the design is concern. The relation with the confinement loss and "effective refractive index" is given by [13],

$$
C . \mathbf{L}=\frac{20}{\ln (10)} \times \frac{2 \pi}{\lambda} \times I M\left(n_{\text {eff }}\right)
$$

The CL or confinement loss is expressed in the form of $\mathrm{dB} / \mathrm{m}$. Here, $I M\left(n_{\text {eff }}\right)$ refers the imaginary part of the complex effective index of refraction and " $\lambda$ "refers to the wavelength of light.

\subsection{Index-Matched Resonant Coupling}

For the purpose of suppressing the HOMs (higher order modes), the index matching resonant coupling is the most popular method where the core-HOM has been indexmatched with that of the cladding leaky defect modes.

The schematic representation of the higher order mode suppression method based on the indexmatching resonant coupling is represented in Fig-1.

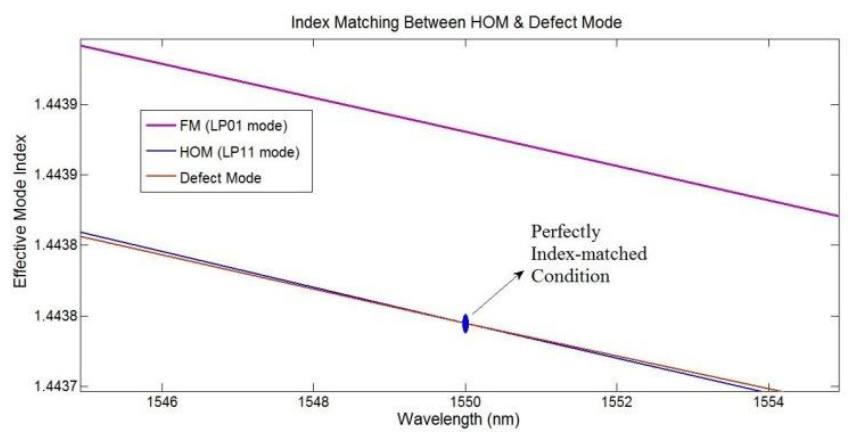

Fig-1: Index matching (at $1.55 \mu \mathrm{m}$ ) of the unwanted coreHOM and the cladding defect modes

\section{PCF DESIGN \& PARAMETERS}

The crosssection design of the optimised LMA photonic crystal fiber is depicted in Fig-1. The fiber has the seven missing air-holes in the center, which create the core region 
and four defects containing small air-holes present in the cladding region. Moreover, the core-region is surrounded by total five air-hole rings subsequently.

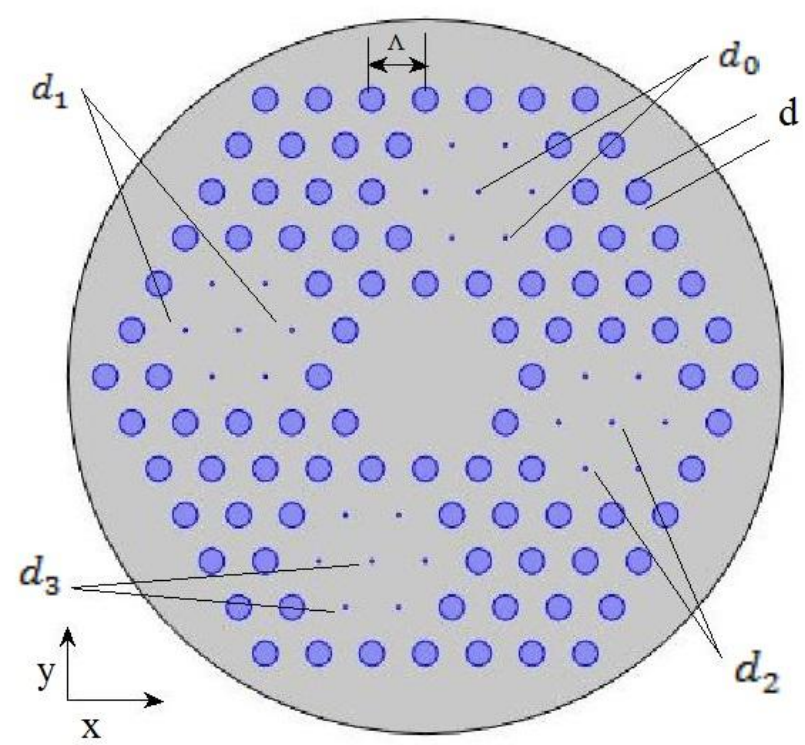

Fig-2: Cross-section design of the "LMA PCF" with defects formed by corresponding small air holes

Here, $\Lambda$ is the holepitch, $d$ refers to the diameter of the cladding air holes and $d_{0}-d_{3}$ are the diameters of the defects containing small air-holes respectively. In order to design the broadband LMA PCF, the proposed design has been tested for four different sub-windows of wavelengths $1.5(\mu \mathrm{m})$ to $1.54(\mu \mathrm{m}), 1.53(\mu \mathrm{m})$ to $1.57(\mu \mathrm{m}), 1.56(\mu \mathrm{m})$ to $1.6(\mu \mathrm{m})$ and $1.59(\mu \mathrm{m})$ to $1.63(\mu \mathrm{m})$ for corresponding defect modes with hole-diameters $d_{0}, d_{1}, d_{2}$ and $d_{3}$ respectively.

It is found that for fibers with $\mathrm{d} / \Lambda<0.41$ are endlessly single-moded for all wavelengths and the fibers with $d / \Lambda>$ 0.45 can be multimoded [14]. Therefore, the proposed design, which has $\mathrm{d} / \Lambda=0.458$, needs to be optimised so that the fiber behaves like a single-moded fiber. Because of the diameters of the air holes in the defect regions are lesser than that of cladding air-holes, the defect modes are tend to leak away. The value of $d_{0}-d_{3}$ can be optimised such that the effective mode index of that leaky defect-mode can match the effective mode index of that "unwanted HOM" at the desired wavelengths. Optimised values of $d_{0}-d_{3}$ are shown in Table 1.

The "effective mode index" of the fiber is calculated by solving the Eigen-value equations using commerciallyavailable software package COMSOL Multiphysics. In this case,FEM ("finite element method") has been used. MATLAB-Live link with COMSOL Multiphysics is used to get the background index of refraction (n), which is calculated by using the Sellmeier's equation for silica [15].

$n^{2}-1=\sum_{i=1}^{3} \frac{A_{i} \cdot \lambda^{2}}{\lambda^{2}-l_{i}^{2}}$
Here, " $n$ " is the index of refraction of the pure fused silica, $A_{i}$ and $l_{i}$ are the Sellmeier's co-efficients. Which are: $A_{1}$ $=0.6961663, l_{1}=6.84043, A_{2}=0.4079426, l_{2}=0.11624140, A_{3}$ $=0.89747940$ and $l_{3}=9.8961610$

Table-1: Optimised Parameters for PCF Design

\begin{tabular}{|c|c|c|c|c|c|c|c|}
\hline $\begin{array}{c}\text { Param } \\
\text { eters }\end{array}$ & $\mathrm{d}$ & $\Lambda$ & $\mathrm{d} / \Lambda$ & $\boldsymbol{d}_{\mathbf{0}}$ & $\boldsymbol{d}_{\mathbf{1}}$ & $\boldsymbol{d}_{\mathbf{2}}$ & $\boldsymbol{d}_{\mathbf{3}}$ \\
\hline Value( & 1 & 21. & 0.458 & 1.47 & 1.47 & 1.487 & 1.4 \\
$\mu \mathrm{m})$ & 0 & 8 & 715 & 738 & 22 & 6 & 817 \\
\hline
\end{tabular}

\section{METHOD OF ANALYSIS}

In order todesign and analysis the fiber in COMSOL Multiphysics, the following are followed in proper order:

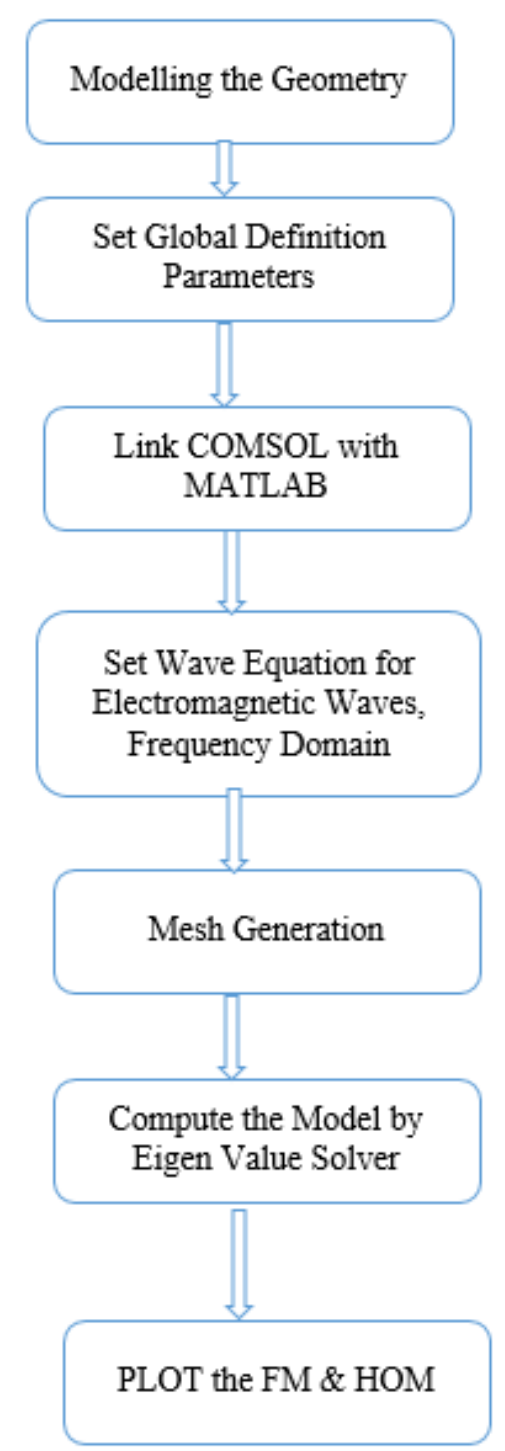

Fig-3: The flowchart for modelling and analysis of LMA photonic crystal fiber in COMSOL-Multiphysics with MATLAB-livelink. 
In first step, the proposed PCF having hexagonal geometry is designed with appropriate geometrical parameters. Thereafter, the Global Definition Parameters viz.the wavelength of light used and air hole-size \& pitch size are set.

Then using MATLAB live-link; refractive index of silica is given off using the "Sellmeier's equation no-(10)" [15]. Then the mesh generation has been done for refinement. The "effective mode index" of the fiber is then computed by resolving the Eigen-value equations using COMSOL Multiphysics' finite element method (FEM). Finally, all of the results are illustrated using generation of Surface and as well as contour plots.

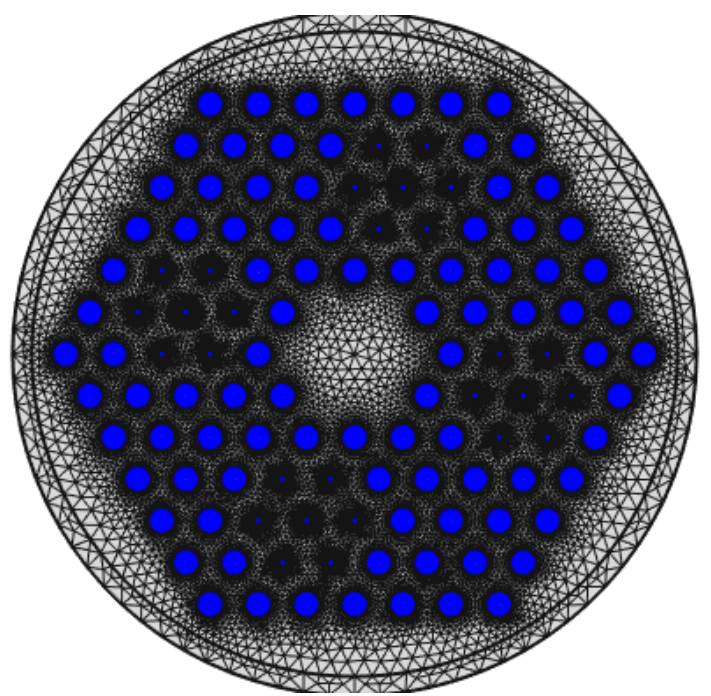

Fig- 4: Generation of Mesh

\section{RESULTS AND DISCUSSION}

For the purpose of finding the index-matching resonant coupling condition, the first thing is to calculate the effective refractive index for the defect modes (containing small air holes) at different wavelength $1.52(\mu \mathrm{m}), 1.55(\mu \mathrm{m})$, $1.58(\mu \mathrm{m})$ and $1.61(\mu \mathrm{m})$ respectively.

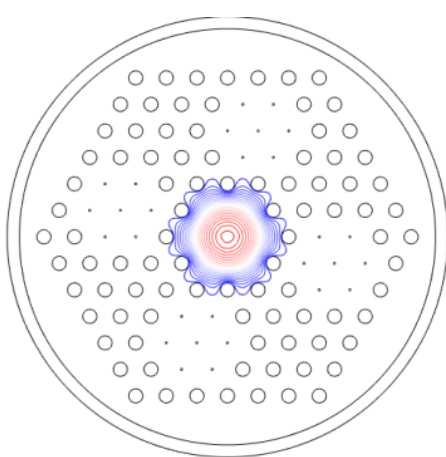

(a)

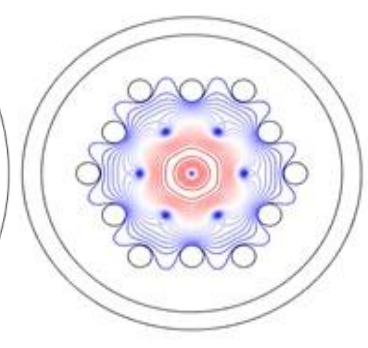

(b)
Fig-5:The Contour-plot of the modal power distribution: (a)

Good confinement of FM for LMA PCF and (b) indexmatched condition of defect modes
The targets for the LMA design are to attain a better confinement for the fundamental mode (loss below $0.01 \mathrm{~dB} / \mathrm{m}$ ) and to reveal higher loss for higher order modes.

Because of the diameters of the air holes in the defect regions are lesser than that of cladding air-holes, the defect modes are tends to leak away.

Fortunately, the coupling-strength between the defect modes and that of the unwanted core higher order modes (HOMs) increases with the decrement of $d_{0}-d_{3}$.

When core higher order modes and cladding defect modes are perfectly index-matched, the power is being mixed betweenthis two regions i.e. at resonant coupling, power is present both in core and cladding.

- Index-matching Resonant Coupling for $1520 \mathrm{~nm}$ subwindow:

1.52e-6 Effective mode index $=1.444164+4.120546 \mathrm{e}-8 \mathrm{i}$ Surface: Electric field, $x$ component $(\mathrm{V} / \mathrm{m})$

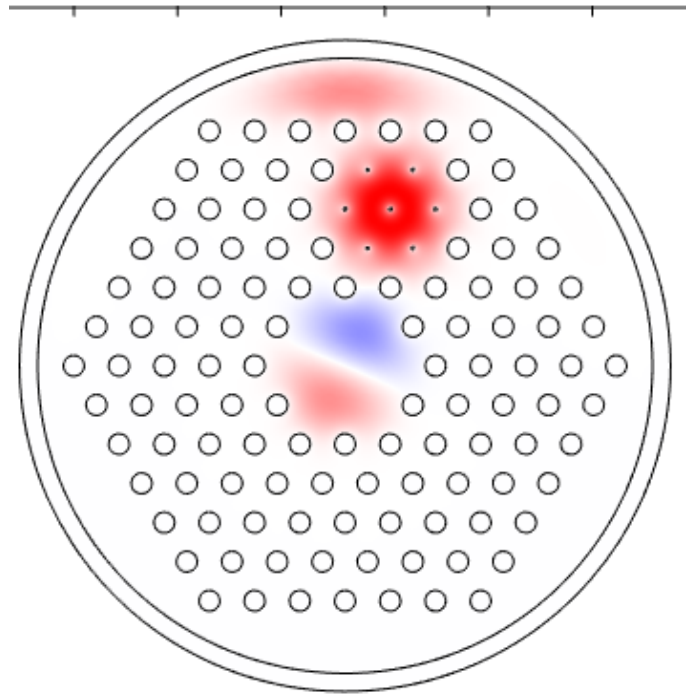

(a)

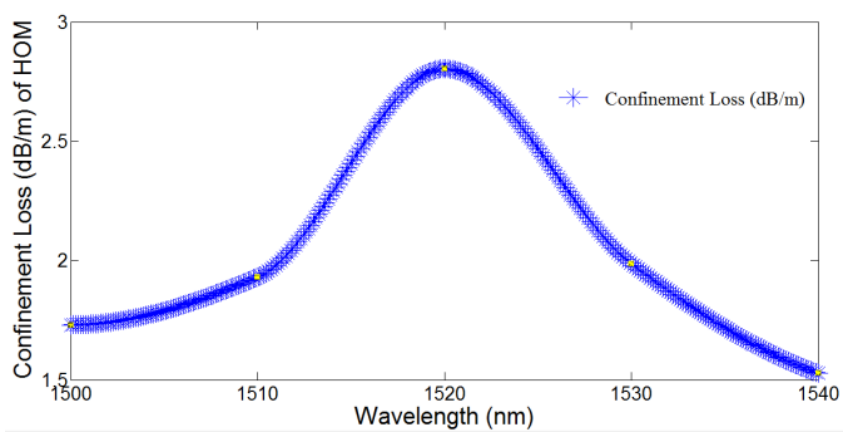

(b)

Fig-6: (a) Leaking of HOM by index-matching resonant coupling for $1.52 \mu \mathrm{m}$ sub-window

(b) Confinement losses of core-HOM after resonant coupling for $1.52 \mu \mathrm{m}$ sub-window 
- Index-matching Resonant Coupling for 1550 nm subwindow:

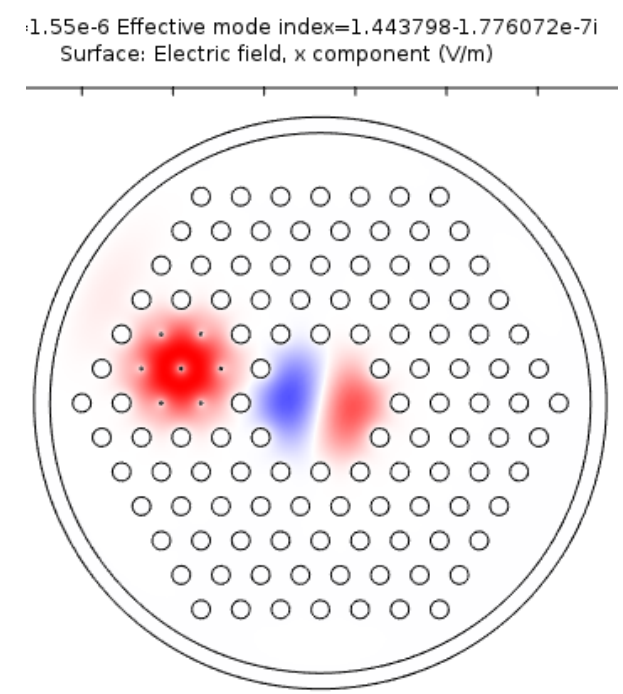

(a)

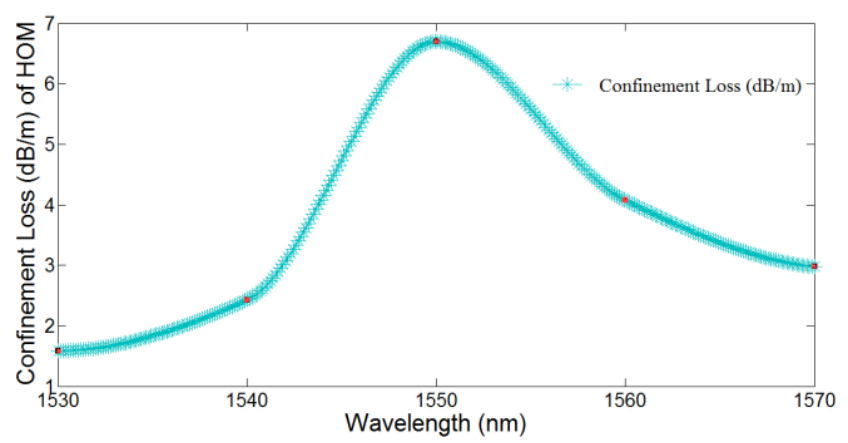

(b)

Fig-7: (a) Leaking of HOM by index-matching resonant coupling for $1.55 \mu \mathrm{m}$ sub-window

(b) Confinement losses of core-HOM after resonant coupling for $1.55 \mu \mathrm{m}$ sub-window

- Index-matching Resonant Coupling for $1580 \mathrm{~nm}$ subwindow:

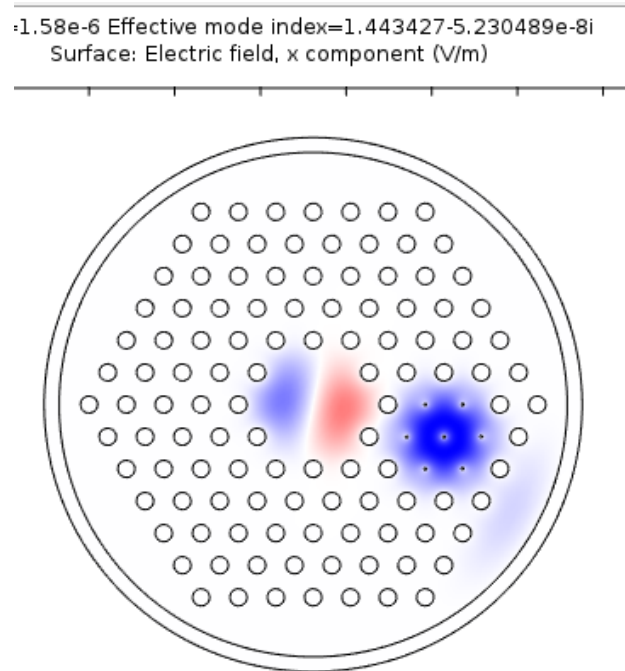

(a)

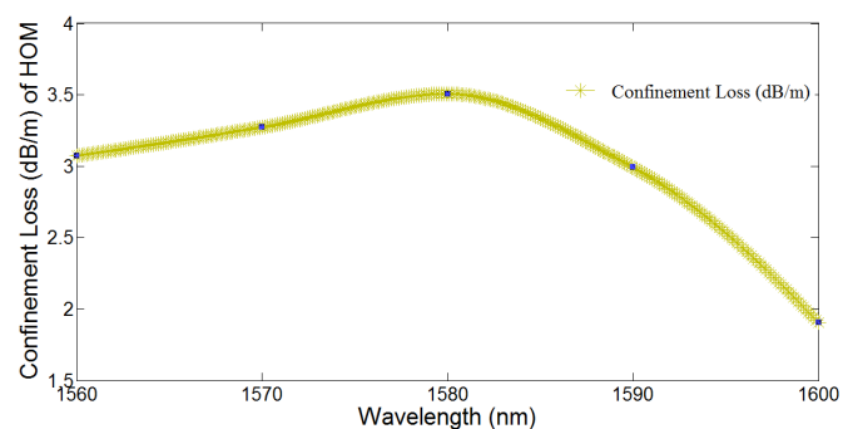

(b)

Fig-8: (a) Leaking of HOM by index-matching resonant coupling for $1.58 \mu \mathrm{m}$ sub-window

(b) Confinement losses of core-HOM after resonant coupling for $1.58 \mu \mathrm{m}$ sub-window

- Index-matching Resonant Coupling for $1610 \mathrm{~nm}$ subwindow:

\subsection{1 e-6 Effective mode index $=1.443053-4.581452 \mathrm{e}-8 \mathrm{i}$ Surface: Electric field, $x$ component $(\mathrm{V} / \mathrm{m})$}

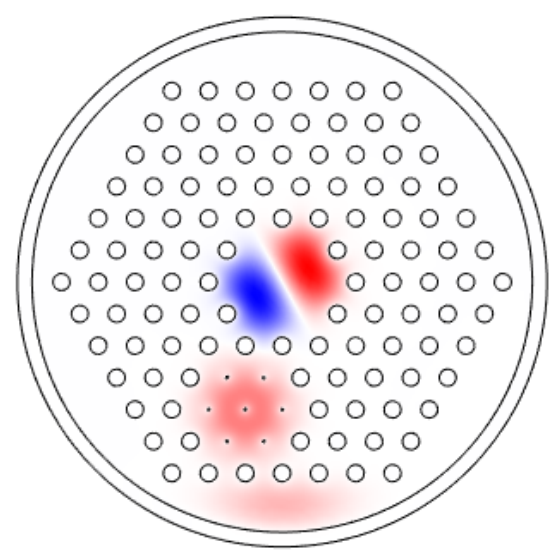

(a)

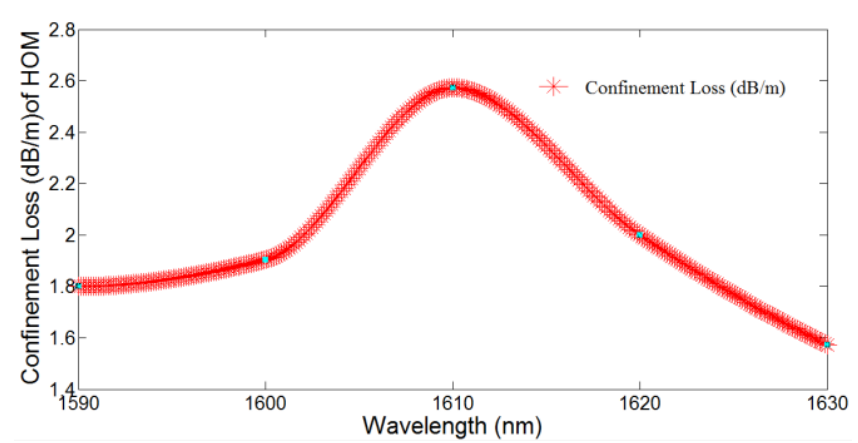

(b)

Fig-9: (a) Leaking of HOM by index-matching resonant coupling for $1.61 \mu \mathrm{m}$ sub-window

(b) Confinement losses of core-HOM after resonant coupling for $1.61 \mu \mathrm{m}$ sub-window

Again, in order to witness the wideband performance of this proposed fiber, further we calculated the leakage or confinement losses of the HOM (LP11 mode) over a wide range of wavelength based on FEM. 
As the figure-(6) to (10) show, the role of these cladding defects have a strong impact on the confinement-loss curves. We also can see, the largest confinement or leakage loss occurs where thesecore-HOMs of LMA fiber and defect modes are index matched.

\section{- Index-matching Resonant Coupling and confinement} loss plot while all the defects are presents :

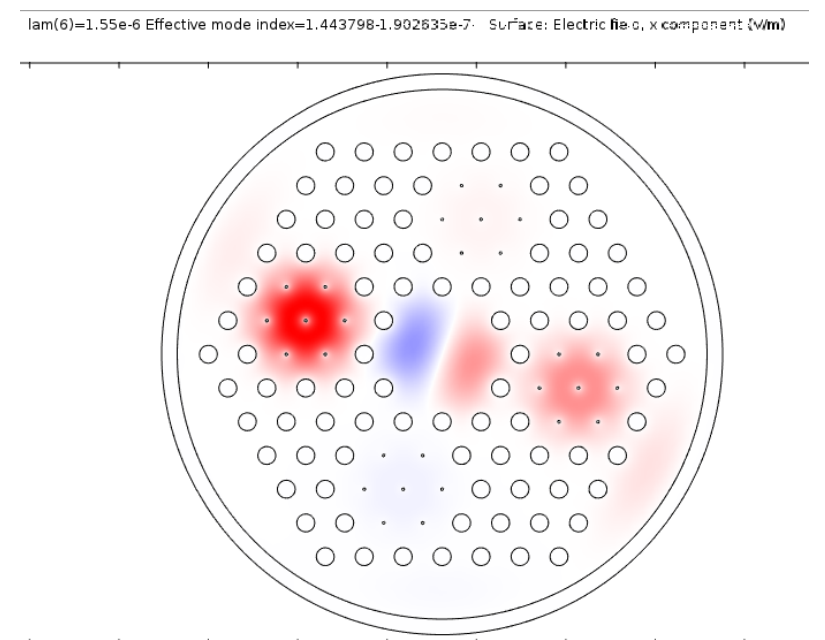

(a)

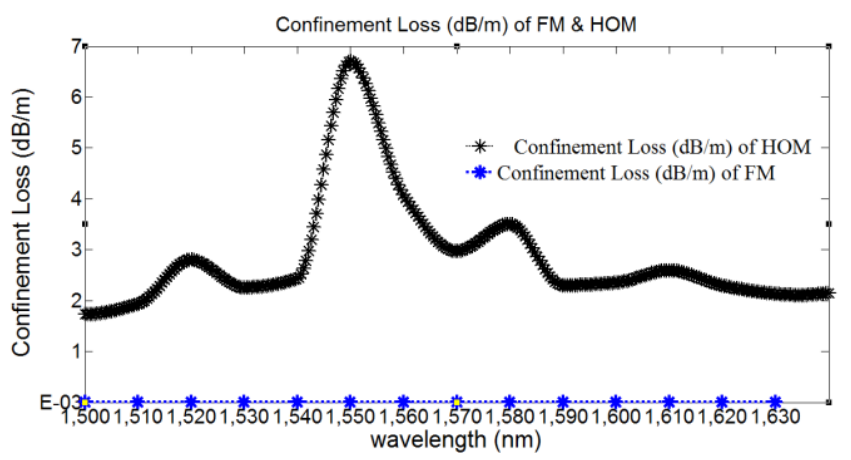

(b)

Fig-10: (a) Leaking of HOM by index-matching resonant coupling when all the defects are presents

(b) Confinement losses of core-FM and HOM after resonant coupling for entire bandwidth

From Fig.6-10, we also can see that in the range of wavelength $1.5 \mu \mathrm{m}$ to $1.63 \mu \mathrm{m}$, core-HOM exhibits the largest leakage or confinement loss of $6.8 \mathrm{~dB} / \mathrm{m}$ in the vicinity of $1.55 \mu \mathrm{m}$ and also the FM leakage loss is below $0.01 \mathrm{~dB} / \mathrm{m}$.

Moreover, when the mode field power is chivied by more than that of $20 \mathrm{~dB}$, then the mode is no longer take into consideration and also if the mode field power is chivied by more than that of $40 \mathrm{~dB}$, the mode doesn't have any existence [13].
Table-2:Summery of the design

\begin{tabular}{|l|l|}
\hline Traits of the optimised LMA PCF & values \\
\hline Mode field area $\left[\mu m^{2}\right]$ & $2200 \mu m^{2}$ \\
\hline FM loss [dB/m] for entire bandwidth & $\begin{array}{l}<10^{-3} \\
\mathrm{~dB} / \mathrm{m}\end{array}$ \\
\hline $\begin{array}{l}\text { Confinement losses [dB/m] of HOM (LP11 } \\
\text { mode) at } 1520 \mathrm{~nm}\end{array}$ & $2.9 \mathrm{~dB} / \mathrm{m}$ \\
\hline $\begin{array}{l}\text { Confinement losses [dB/m] of HOM (LP11 } \\
\text { mode) at } 1550 \mathrm{~nm}\end{array}$ & $6.8 \mathrm{~dB} / \mathrm{m}$ \\
\hline $\begin{array}{l}\text { Confinement losses [dB/m] of HOM (LP11 } \\
\text { mode) at } 1580 \mathrm{~nm}\end{array}$ & $3.5 \mathrm{~dB} / \mathrm{m}$ \\
\hline $\begin{array}{l}\text { Confinement losses [dB/m] of HOM (LP11 } \\
\text { mode) at } 1610 \mathrm{~nm}\end{array}$ & $2.59 \mathrm{~dB} / \mathrm{m}$ \\
\hline
\end{tabular}

\section{CONCLUSIONS}

In this paper, a novel 7 cell-core large mode area (LMA) photonic crystal fiber (PCF)has been presented, which is designed with five rings hexagonal geometry using FEM (finite element method) based on the theory of higher order mode suppression through the indexmatching resonant coupling with leaky cladding defect modes. The optimised micro-structured LMA photonic crystal fiber (PCF) exhibits exceptional features for high power fiber lasersas well as for amplifier applications, along with an effective large MFA (mode field area) around $2200 \mu \mathrm{m}^{2}$ as well as mono-mode operation by suppression of higher order mode. The LMA$\mathrm{PCF}$ performance is attained for a wide range of wavelength $1.5 \mu \mathrm{m}$ to $1.63 \mu \mathrm{m}$. Thus, the operational bandwidth reaches $130 \mathrm{~nm}$. By proper selection of the values of $\mathrm{d} / \Lambda$ and $d_{0}-$ $d_{3}$, the leakage loss is controllable and thus the HOMresonant coupling loss is larger than $6.8 \mathrm{~dB} / \mathrm{m}$ at $1.55 \mu \mathrm{m}$. Moreover, the proposed fiber also exhibits FM confinementloss lower than $0.01 \mathrm{~dB} / \mathrm{m}$

Thus, the fiber offers worthy features that allow for the mono-mode operation. The large mode field area can reduce optical intensities and thus the fiber design can gain more attraction in the field of fiber laser and amplifier based applications. Moreover, the broadband design which covers the key wavelength $(1550 \mathrm{~nm})$ for communication window, will pay attention to the manufacturer in the fiber optic communication.

\section{REFERENCES}

[1]. Russell, P. St. J., "Photonic crystal fibers," Science, Vol. 299, 358\{362, 2003.

[2]. M. Napierała, T. Nasiłowski, E. Bereś-Pawlik, F. Berghmans, J. Wójcik, and H. Thienpont, "Extremely largemode-area photonic crystal fiber with low bending loss", Opt. Express 18(15), 15408-15418 (2010).

[3]. X. Wang, S. Lou and W. Lu, "Bending orientation insensitive large mode area photonic crystal fiber with triangular core", IEEE Photon. J., vol. 5, no. 4, pp. 7100408$7100408,2013$. 
[4]. P. Geng, W. Zhang, H. Zhang, S. Zhang, J. Ruan, S. Wei, and X. Xue, "Design of Broadband Single-Polarization Single-Mode Photonic Crystal Fiber Based on IndexMatching Coupling," IEEE Photon. Technol. Lett. 24(6), 452-454 (2012)

[5]. P. G. Geng, W. G. Zhang, S. C. Gao, S. S. Zhang, H. Zhang and J. Ruan, "Orthogonal single-polarization singlecore photonic crystal fiber for wavelength splitting", IEEE Photon. Technol. Lett., vol. 24, no. 15, pp. 1304-1306, 2012

[6].Tadashi Murao, KunimasaSaitoh, and Masanori Koshiba, "Multiple resonant coupling mechanism for suppression of higher-order modes in all-solid photonic bandgap fibers with heterostructured cladding," Opt. Express 19, 1713-1727 (2011)

[7]. Corbett, Jason CW. (2006) "Photonic crystal fibres in astronomy", Durham theses, Durham University. Ph.D. thesis; Dept. of Physics, Durham University.

[8]. Ana M. R. Pinto and Manuel Lopez-Amo, "Photonic Crystal Fibers for Sensing Applications", Hindawi Publishing Corporation Journal of Sensors Volume 2012, Article ID 598178.

[9]. A book on "Silica Optical Fiber Technology for Devices and Components: Design, Fabrication, and International Standards" by Kyunghwan Oh, Un-ChulPaek 'John Wiley \& Sons, 07-Nov-2012 - Technology \& Engineering.

[10]. J. C. Knight, T. A. Birks, P. S. J. Russell, and D. M. Atkin, "Allsilica single-mode optical fiber with photonic crystal cladding" Optics Letters 21, 1547 (1996).

[11]. S.M. ABDUR RAZZAK \& YOSHINORI NAMIHIRA "Theoretical design of a large effective mode area microstructure optical fiber", OpticaApplicata, Vol. XL, No. 3,2010 .

[12]. Aparna A Nair, S.K Sudheer, M. Jayaraju "Analysis of Optical Characteristics for Photonic Crystal Fiber at Small Core Diameters", International Journal of Engineering and Advanced Technology (IJEAT), V/olume-3, Issue-4, April 2014.

[13]. Ming-Yang Chen and Yong-Kang Zhang "Improved design of polarization-maintaining photonic crystal fibers, OPTICS LETTERS / Vol. 33, No. 21 / November 1, 2008, (C) 2008 Optical Society of America.

[14]. K. Saitoh, Y. Tsuchida, M. Koshiba, and N. A. Mortensen, "Endlessly single-mode holey fibers: the influence of core design," Opt. Express 13(26), 1083310839 (2005).

[15]. James W. Fleming, "Dispersion in $\mathrm{GeO} 2-\mathrm{SiO} 2$ glasses," Appl. Opt. 23. pp. 4486-4493 (1984). 